\title{
EXISTENCE OF SUPERCRITICAL PASTING ARCS FOR TWO SHEETED SPHERES
}

\author{
Mitsuru NAKaI
}

\begin{abstract}
Take e.g. two disjoint nondegenerate compact continua $A$ and $B$ in the complex plane $\mathbf{C}$ with connected complements and pick a simple $\operatorname{arc} \gamma$ in the complex sphere $\hat{\mathbf{C}}$ disjoint from $A \cup B$, which we call a pasting arc for $A$ and $B$. Construct a covering Riemann surface $\hat{\mathbf{C}}_{\gamma}$ over $\hat{\mathbf{C}}$ by pasting two copies of $\hat{\mathbf{C}} \backslash \gamma$ crosswise along $\gamma$. We embed $A$ in one sheet and $B$ in another sheet of two sheets of $\hat{\mathbf{C}}_{\gamma}$ which are copies of $\hat{\mathbf{C}} \backslash \gamma$ so that $\hat{\mathbf{C}}_{\gamma} \backslash A \cup B$ is understood as being obtained by pasting $(\hat{\mathbf{C}} \backslash A) \backslash \gamma$ with $(\hat{\mathbf{C}} \backslash B) \backslash \gamma$ crosswise along $\gamma$. In the comparison of the variational 2 capacity $\operatorname{cap}\left(A, \hat{\mathbf{C}}_{\gamma} \backslash B\right)$ of the compact set $A$ considered in the open set $\hat{\mathbf{C}}_{\gamma} \backslash B$ with the corresponding $\operatorname{cap}(A, \hat{\mathbf{C}} \backslash B)$, we say that the pasting arc $\gamma$ for $A$ and $B$ is subcritical, critical, or supercritical according as $\operatorname{cap}\left(A, \hat{\mathbf{C}}_{\gamma} \backslash B\right)$ is less than, equal to, or greater than $\operatorname{cap}(A, \hat{\mathbf{C}} \backslash B)$, respectively. We have shown in our former paper [4] the existence of pasting arc $\gamma$ of any one of the above three types but that of supercritical and critical type was only shown under the additional requirment on $A$ and $B$ that $A$ and $B$ are symmetric about a common straight line simultaneously. The purpose of the present paper is to show that in the above mentioned result the additional symmetry assumption is redundant: we will show the existence of supercritical and hence of critical arc $\gamma$ starting from an arbitrarily given point in $\hat{\mathbf{C}} \backslash A \cup B$ for any general admissible pair of $A$ and $B$ without any further requirment whatsoever.
\end{abstract}

\section{Introduction}

A nonempty compact subset $A$ of the complex plane $\mathbf{C}$ will be referred to as an admissible compact subset if $\hat{\mathbf{C}} \backslash A$ is a regular subregion of the complex sphere $\hat{\mathbf{C}}=\mathbf{C} \cup\{\infty\}$, i.e. a relatively compact and connected open subset of $\hat{\mathbf{C}}$ whose relative boundary $\partial(\hat{\mathbf{C}} \backslash A)$ consists of a finite number of disjoint analytic Jordan curves. Thus an admissible $A$ may or may not be connected and in general it consists of a finite number of connected components which themselves are also admissible. If $B$ is another admissible compact subset of $\mathbf{C}$ disjoint from $A$, then $A \cup B$ is again admissible. For a pair of two disjoint admissible compact subsets

2000 Mathematics Subject Classification. Primary 31A15, 31C15; Secondary 30C85, 30F15.

Key words and phrases. capacity, covering surface, Dirichlet integral, Dirichlet principle, harmonic measure, modulus, two sheeted plane (sphere).

Received May 26, 2005; revised September 14, 2005. 
$A$ and $B$ in $\mathbf{C}$, a simple arc $\gamma$ in $\hat{\mathbf{C}} \backslash A \cup B$ will be referred to as a pasting arc for $A$ and $B$ since we will paste $(\hat{\mathbf{C}} \backslash A) \backslash \gamma$ with $(\hat{\mathbf{C}} \backslash B) \backslash \gamma$ crosswise along $\gamma$. In general consider two subregions $R$ and $S$ in $\hat{\mathbf{C}}$ and a simple arc $\gamma$ in $R \cap S$. We will use (cf. [5]) the convenient notation $(R \backslash \gamma) \bigotimes_{\gamma}(S \backslash \gamma)$ for the Riemann surface obtained from $R$ and $S$ by pasting $R \backslash \gamma$ with $S \backslash \gamma$ crosswise along $\gamma$. For a pair of two disjoint admissible compact subsets $A$ and $B$ in $\mathbf{C}$ and a pasting arc $\gamma$ for $A$ and $B$ we will consider a new Riemann surface

$$
\hat{\mathbf{C}}_{\gamma}:=(\hat{\mathbf{C}} \backslash \gamma) \bigotimes_{\gamma}(\hat{\mathbf{C}} \backslash \gamma)
$$

and also its subsurface

$$
W_{\gamma}:=\hat{\mathbf{C}}_{\gamma} \backslash A \cup B
$$

where we understand that $A$ (B, resp.) is embedded in the upper (lower, resp.) sheet $\hat{\mathbf{C}} \backslash \gamma$ of $\hat{\mathbf{C}}_{\gamma}$ although $A$ and $B$ are originally contained in the same $\mathbf{C}$. Hence

$$
W_{\gamma}=((\hat{\mathbf{C}} \backslash A) \backslash \gamma) \bigotimes_{\gamma}((\hat{\mathbf{C}} \backslash B) \backslash \gamma) .
$$

Here $\hat{\mathbf{C}}_{\gamma}$ is a covering Riemann surface $\left(\hat{\mathbf{C}}_{\gamma}, \hat{\mathbf{C}}, \pi_{\gamma}\right)$ of the base surface $\hat{\mathbf{C}}$ with the natural projection $\pi_{\gamma}$.

Consider next the capacity $\operatorname{cap}\left(A, \hat{\mathbf{C}}_{\gamma} \backslash B\right)$, or more precisely the variational 2 capacity (cf. e.g. [2]), of the compact subset $A$ in $\hat{\mathbf{C}}_{\gamma}$ with respect to the open subset $\hat{\mathbf{C}}_{\gamma} \backslash B$ of $\hat{\mathbf{C}}_{\gamma}$ containing $A$ given by

$$
\operatorname{cap}\left(A, \hat{\mathbf{C}}_{\gamma} \backslash B\right):=\inf _{\varphi} D\left(\varphi ; W_{\gamma}\right),
$$

where $\varphi$ in taking the infimum in (1.3) runs over the family of $\varphi \in C\left(\hat{\mathbf{C}}_{\gamma}\right) \cap$ $C^{\infty}\left(W_{\gamma}\right)$ with $\varphi \mid A=1$ and $\varphi \mid B=0$ and $D\left(\varphi ; W_{\gamma}\right)$ indicates the Dirichlet integral of $\varphi$ over $W_{\gamma}$ defined by

$$
D\left(\varphi ; W_{\gamma}\right):=\int_{W_{\gamma}} d \varphi \wedge * d \varphi=\int_{W_{\gamma}}|\nabla \varphi(z)|^{2} d x d y .
$$

Here the second term in the above is the coordinate free expression of $D\left(\varphi, W_{\gamma}\right)$ and the third term is the expression of $D\left(\varphi, W_{\gamma}\right)$ in terms of local parameters $z=x+i y$ for $W_{\gamma}$ and $\nabla \varphi(z)$ is the gradient vector $(\partial \varphi(z) / \partial x, \partial \varphi(z) / \partial y)$. Clealy we have the following symmetry:

$$
\operatorname{cap}\left(A, \hat{\mathbf{C}}_{\gamma} \backslash B\right)=\operatorname{cap}\left(B, \hat{\mathbf{C}}_{\gamma} \backslash A\right) .
$$

The variation (1.3) has the unique extremal function $u_{\gamma}$ :

$$
\operatorname{cap}\left(A, \hat{\mathbf{C}}_{\gamma} \backslash B\right)=D\left(u_{\gamma} ; W_{\gamma}\right),
$$

characterized by the conditions $u_{\gamma} \in C\left(\hat{\mathbf{C}}_{\gamma}\right) \cap H\left(W_{\gamma}\right)$ with $u_{\gamma} \mid A=1$ and $u_{\gamma} \mid B=0$ (cf. e.g. [2]), where $H(X)$ denotes the class of harmonic functions defined on an open subset $X$ of a Riemann surface so that the function $u_{\gamma} \mid W_{\gamma}$ is usually referred to as the harmonic measure of $A \cap \partial W_{\gamma}$ (cf. e.g. [8]). The extremal function $u_{\gamma}$ for (1.3) is also referred to as the capacity function for the compact subset $A$ with respect to $\hat{\mathbf{C}}_{\gamma} \backslash B$ (cf. [7]). 
We also consider the capacity $\operatorname{cap}(A, \hat{\mathbf{C}} \backslash B)$ of the subset $A$ in $\hat{\mathbf{C}}$ contained in the open subset $\hat{\mathbf{C}} \backslash B$. Similarly as in the case of $\operatorname{cap}\left(A, \hat{\mathbf{C}}_{\gamma} \backslash B\right)$ we have the $\operatorname{symmetry} \operatorname{cap}(A, \hat{\mathbf{C}} \backslash B)=\operatorname{cap}(B, \hat{\mathbf{C}} \backslash A)$ and that the capacity cap $(A, \hat{\mathbf{C}} \backslash B)$ is given by the capacity function $u$ for the compact subset $A$ with respect to $\hat{\mathbf{C}} \backslash B$ :

$$
\operatorname{cap}(A, \hat{\mathbf{C}} \backslash B)=D(u ; W) \quad(W:=\hat{\mathbf{C}} \backslash A \cup B),
$$

where $u \in C(\hat{\mathbf{C}}) \cap H(W)$ with $u \mid A=1$ and $u \mid B=0$. Motivated by the problem to clarify when the situation $\operatorname{cap}\left(A, \hat{\mathbf{C}}_{\gamma} \backslash B\right) \leq \operatorname{cap}(A, \hat{\mathbf{C}} \backslash B)$ holds, which occured in the study of the classical and modern type problem (cf. e.g. [6], [10], [8], [5], [3], among many others), the following classification problem of pasting arcs started: since the occurence of

$$
\operatorname{cap}\left(A, \hat{\mathbf{C}}_{\gamma} \backslash B\right)=\operatorname{cap}(A, \hat{\mathbf{C}} \backslash B)
$$

is very delicate in the sense that the relation is easily destroyed even if we change $\gamma$ slightly, the pasting arc $\gamma$ for $\hat{\mathbf{C}}_{\gamma}$ for which we have (1.5) is referred to as being critical. In contrast the situation

$$
\operatorname{cap}\left(A, \hat{\mathbf{C}}_{\gamma} \backslash B\right)<\operatorname{cap}(A, \hat{\mathbf{C}} \backslash B)
$$

and also the situation

$$
\operatorname{cap}\left(A, \hat{\mathbf{C}}_{\gamma} \backslash B\right)>\operatorname{cap}(A, \hat{\mathbf{C}} \backslash B)
$$

are quite stable with respect to the small perturbation of $\gamma$ and the pasting arc $\gamma$ for which the relation (1.6) ((1.7), resp.) holds is referred to as being subcritical (supercritical, resp.). The occurence for a pasting arc $\gamma$ to be subcritical is just very common. For example, if the daiameter of $\gamma$ is sufficiently small, then $\gamma$ is subcritical (cf. [4]). In view of this it was expected in one time that every pasting arc $\gamma$ satisfies (1.5) or (1.6) and there is no $\gamma$ for which (1.7) is valid. We found, however, (1.7) can really occur in our former paper [4] when the pair of disjoint admissible compact subsets $A$ and $B$ are symmetric with respect to a common straight line. The purpose of the present paper is to show that without any additional condition the above is correct: for any pair of disjoint admissible compact subsets $A$ and $B$ in $\mathbf{C}$ there always exists a supercritical pasting arc $\gamma$ for $A$ and $B$. Hence we have

THEOREM. For any pair of disjoint admissible compact subsets $A$ and $B$ of $\mathbf{C}$, there always exist pasting arcs $\gamma_{1}, \gamma_{2}$, and $\gamma_{3}$ in $\mathbf{C}$ for $A$ and $B$ starting from an arbitrarily given nonsingular point $a$ in $\mathbf{C} \backslash A \cup B$ of the gradient of the capacity function on $\hat{\mathbf{C}}$ for $A$ and $B$ such that $\gamma_{1}$ is critical, $\gamma_{2}$ is subcritical, and $\gamma_{3}$ is supercritical.

Not only the mere existence but also the criteria for a given pasting arc $\gamma$ to be subcrical are discussed in detail in [4]. For a pasting arc $\gamma$ starting from a point $a \in \mathbf{C}$, we denote by $\gamma_{z}$ for any $z \in \gamma \backslash\{a\}$ the subarc of $\gamma$ starting from $a$ and terminating at $z$. We have also shown in [4] that if $\gamma$ is supercritical, then there are points $s$ and $c$ in $\gamma \backslash\{a\}$ such that $\gamma_{s}\left(\gamma_{c}\right.$, resp.) is subcritical (critical, resp.). Hence to complete the proof of the above theorem, we only have to show the existence of a supercritical arc, which is the actual work in this paper. 


\section{Proof of the theorem}

As mentioned in the introduction we only have to prove the existence of a supercritical pasting arc $\gamma$ for an arbitrarily given general pair of disjoint admissible compact subsets $A$ and $B$ in the complex plane C. We set

$$
W:=\hat{\mathbf{C}} \backslash(A \cup B) .
$$

We denote by $u$ the capacity function on $\hat{\mathbf{C}}$ for the capacity of $A$ with respect to $\hat{\mathbf{C}} \backslash B$ :

$$
\operatorname{cap}(A, \hat{\mathbf{C}} \backslash B)=D(u ; \hat{\mathbf{C}})
$$

so that $u \in C(\hat{\mathbf{C}}) \cap H(\hat{\mathbf{C}} \backslash A \cup B), u \mid A=1$, and $u \mid B=0$. Hence $u \mid W$ is the harmonic measure of $A \cap \partial W$ on $W$. Choose an arbitrary but then fixed nonsingular point $a \in \hat{\mathbf{C}} \backslash A \cup B$ of the gradient vector field of $u: d u(a) \neq 0$. There is an arc $l$ containing $a$ as its interior point, on which $d u \neq 0$, such that

$$
* d u=0
$$

along $l$, i.e. $l$ is a $u$ conjugate level arc. We pick an arbitrary interior point $b$ in $l$ other than $a$ such that $u(z)$ decreases as $z$ traces $l$ from $a$ to $b$. We take an arbitrary but then fixed smooth Jordan curve $\sigma$ encircling $B$ and intersecting with $l$ only once at $b$. We give a negative direction to $\sigma$. We denote by $(\sigma)$ the region bounded by $\sigma$. Then $\bar{B} \subset(\sigma)$ and $a$ is an interior point in the arc $l \backslash(\sigma)$. Then consider the subarc $\tau$ of $l$ whose initial point is $a$ and the terminal point is $b$. Thus $\tau$ is a $u$ conjugate level arc with the positive direction starting from $a$ and ending at $b$. In general we denote by $|\gamma|$ the length of an arc $\gamma$ measured by the plane metric. For each $t \in(0,|\sigma| / 100)$ we pick the point $c(t) \in \sigma$ such that the subarc $\sigma_{t}^{\prime}$ of $\sigma$ starting from $c(t)$ and ending at $b$ in the direction of $\sigma$ satisfies $\left|\sigma_{t}^{\prime}\right|=t$. We then put $\sigma_{t}:=\overline{\sigma \backslash \sigma_{t}^{\prime}}$, the subarc of $\sigma$ starting from $b$ and ending at $c(t)$ in the direction of $\sigma$. Finally we consider the arc

$$
\gamma_{t}:=\tau+\sigma_{t} \quad(0<t<|\sigma| / 100) .
$$

Here 100 in (2.4) has no particular meaning other than suggesting the point $c(t)$ is situated enough close to the point $b$ since we are making $t \downarrow 0$ later.

We will show that $\gamma_{t}$ in (2.4) is a supercritical arc if we choose $t \in(0,|\sigma| / 100)$ sufficiently small:

$$
\operatorname{cap}\left(A, \hat{\mathbf{C}}_{\gamma_{t}} \backslash B\right)>\operatorname{cap}(A, \hat{\mathbf{C}} \backslash B)
$$

for sufficiently small $t \in(0,|\sigma| / 100)$. For simplicity we set

$$
W_{t}:=W_{\gamma_{t}}=\hat{\mathbf{C}}_{\gamma_{t}} \backslash A \cup B=\left((\hat{\mathbf{C}} \backslash A) \backslash \gamma_{t}\right) \bigotimes_{\gamma_{t}}\left((\hat{\mathbf{C}} \backslash B) \backslash \gamma_{t}\right)
$$

and also we denote by $u_{t}:=u_{\gamma_{t}}$, the capacity function on $\hat{\mathbf{C}}_{\gamma_{t}}$ for $\operatorname{cap}\left(A, \hat{\mathbf{C}}_{\gamma_{t}} \backslash B\right)$ so that $u_{t} \in C\left(\hat{\mathbf{C}}_{\gamma_{t}}\right) \cap H\left(W_{t}\right)$ with $u_{t} \mid A=1$ and $u_{t} \mid B=0$. We also consider an auxiliary surface

$$
W_{0}:=((\hat{\mathbf{C}} \backslash A \cup B) \backslash \tau)\left(\bigotimes_{\tau}(\hat{\mathbf{C}} \backslash \tau)\right.
$$


We denote by $\delta_{t}\left(\delta_{0 t}\right.$, resp.) the part of $W_{t}\left(W_{0}\right.$, resp.) lying over $\sigma_{t}^{\prime}=\overline{\sigma \backslash \sigma_{t}}$, which consists of two copies of $\sigma_{t}^{\prime}$ situated in each of two sheets of $\hat{\mathbf{C}}_{\gamma_{t}}\left(\hat{\mathbf{C}}_{\tau}\right.$, resp.). Finally we put $W_{t}^{\prime}=W_{t} \backslash \delta_{t}$ and observe the following two and, especially the second, crucial relations in our proof:

$$
W_{t}^{\prime} \subset W_{s}^{\prime} \quad(0<s \leq t<|\sigma| / 100)
$$

and

$$
W_{t}^{\prime}=W_{0} \backslash \delta_{0 t} \quad(0<t<|\sigma| / 100) .
$$

The function $u_{t}$, originally defined on $W_{t}$ so that on $W_{t}^{\prime}$, may also be considered as being defined on $W_{0} \backslash \delta_{0 t}$ by (2.9) but its boundary values at $\delta_{0 t}$ must be considered in the sense of Carathéodory, i.e. a single point in $\delta_{0 t}$ is considered as two boundary elements in the Carathéodory compactification of $W_{0} \backslash \delta_{0 t}$ (cf. [10]). Let $w_{t}$ be the function on $\hat{\mathbf{C}}_{\tau}$ such that $w_{t} \in C\left(\hat{\mathbf{C}}_{\tau}\right) \cap H\left(W_{0} \backslash \delta_{0 t}\right)$ with $w_{t}\left|A=w_{t}\right| B=0$ and $w_{t} \mid \delta_{0 t}=1$. By comparing boundary values we see that $0 \leq w_{s} \leq w_{t} \leq 1 \quad(0<s \leq t)$ on $W_{0} \backslash \delta_{0 t}$. Hence $\left(w_{t}\right)_{t ! 0}$ converges to a function $w \in C\left(\hat{\mathbf{C}}_{\tau} \backslash\{\tilde{b}\}\right) \cap H\left(W_{0} \backslash\{\tilde{b}\}\right)$ with $0 \leq w \leq 1$ on $\hat{\mathbf{C}}_{\tau} \backslash\{\tilde{b}\}$ and $w|A=w| B=0$ almost uniformly on $\hat{\mathbf{C}}_{\tau} \backslash\{\tilde{b}\}$, where $\tilde{b}$ is the branch point of $\hat{\mathbf{C}}_{\tau}$ lying over $b$. By the Riemann removability theorem we see that $w \in H\left(W_{0}\right)$ with boundary values 0 so that $w=0$ and a fortiori

$$
\lim _{t \downarrow 0} w_{t}=0
$$

almost uniformly on $\hat{\mathbf{C}}_{\tau} \backslash\{\tilde{b}\}$. Clearly, by comparing the boundary values, we see that

$$
\left|u_{t}-u_{s}\right| \leq w_{t} \quad(0<s \leq t)
$$

on $\bar{W}_{0} \backslash \delta_{0 t}$ and hence on $\hat{\mathbf{C}}_{\tau} \backslash \delta_{0 t}$. Therefore, by (2.10), we see that $\left(u_{t}\right)_{t \downarrow 0}$ converges to a function $v \in C\left(\hat{\mathbf{C}}_{\tau} \backslash\{\tilde{b}\}\right) \cap H\left(W_{0} \backslash\{\tilde{b}\}\right)$ almost uniformly on $\hat{\mathbf{C}}_{\tau} \backslash\{\tilde{b}\}$ such that $v \mid A=1$ and $v \mid B=0$ and $0 \leq v \leq 1$ on $\hat{\mathbf{C}}_{\tau} \backslash\{\tilde{b}\}$. Again by the Riemann removability theorem, $v \in H\left(W_{0}\right)$ and thus of course $v \in\left(\hat{\mathbf{C}}_{\tau}\right) \cap H\left(W_{0}\right)$.

Set $\alpha:=A \cap \partial W_{0}$ and $\beta:=B \cap \partial W_{0}$. Then we also see that $\alpha=A \cap \partial W_{t}$ and $\beta=B \cap \partial W_{t}$ for any $0<t<|\sigma| / 100$. We give the positive orientation to $\alpha$ and $\beta$ with respect to the region $W_{0}$ and hence to $W_{t}$ for every $0<t<|\sigma| / 100$. Since $\alpha$ and $\beta$ are analytic with $u_{t}|\alpha=v| \alpha=1$ and $u_{t}|\beta=v| \beta=0, u_{t}$ and $v$ are extendable as uniformly bounded harmonic functions to a fixed vicinity of $\alpha \cup \beta$ and $\left(u_{t}\right)_{t \downarrow 0}$ converges uniformly to $v$ there. Hence

$$
\lim _{t \downarrow 0} * d u_{t}=* d v
$$

uniformly on $\alpha \cup \beta$ in the sense that coefficients of $* d u_{t}$ converge uniformly to the corresponding coefficients of $* d v$ in any small parametric disc centered at any point of $\alpha \cup \beta$. Hence in particular we see that

$$
\lim _{t \downarrow 0} \int_{\alpha} * d u_{t}=\int_{\alpha} * d v
$$


Observe that, by the Stokes formula, we see

$$
\operatorname{cap}\left(A, \hat{\mathbf{C}}_{\gamma_{t}} \backslash B\right)=D\left(u_{\gamma_{t}} ; \hat{\mathbf{C}}_{\gamma_{t}}\right)=D\left(u_{\gamma_{t}} ; \hat{\mathbf{C}}_{\gamma_{t}} \backslash A \cup B\right)=\int_{\alpha} * d u_{\gamma_{t}}=\int_{\alpha} * d u_{t} .
$$

Understanding this time that $A$ and $B$ are contained in the same one sheet $\hat{\mathbf{C}} \backslash \tau$ of $\hat{\mathbf{C}}_{\tau}=(\hat{\mathbf{C}} \backslash \tau)\left(\otimes_{\tau}(\hat{\mathbf{C}} \backslash \tau)\right.$ as in the case of $W_{0}$, we compute the capacity $\operatorname{cap}\left(A, W_{0} \cup A\right)$ of the compact subset $A$ of $\hat{\mathbf{C}}_{\tau}$, considered as $\hat{\mathbf{C}}_{\tau}=$ $\left[((\hat{\mathbf{C}} \backslash A) \backslash \tau) \bigotimes \bigotimes_{\tau}(\hat{\mathbf{C}} \backslash \tau)\right] \cup A=W_{0} \cup(A \cup B) \supset A$, with respect to the open subset $W_{0} \cup A=((\hat{\mathbf{C}} \backslash B) \backslash \tau) \bigotimes \bigotimes_{\tau}(\hat{\mathbf{C}} \backslash \tau)$ of $\hat{\mathbf{C}}_{\tau}$ containing $A$. Then, since $v$ is the capacity function for $\operatorname{cap}\left(A, W_{0} \cup A\right)$, by exactly the same argument as above we see that

$$
\operatorname{cap}\left(A, W_{0} \cup A\right)=D\left(v ; \hat{\mathbf{C}}_{\tau}\right)=D\left(v ; \hat{\mathbf{C}}_{\tau} \backslash A \cup B\right)=\int_{\alpha} * d v .
$$

Hence by (2.12) we deduce that

$$
\lim _{t \downarrow 0} \operatorname{cap}\left(A, \hat{\mathbf{C}}_{\gamma_{t}} \backslash B\right)=\operatorname{cap}\left(A, W_{0} \cup A\right) .
$$

Finally we $\operatorname{compare} \operatorname{cap}\left(A, W_{0} \cup A\right)$ with $\operatorname{cap}(A, \hat{\mathbf{C}} \backslash B)$. Recall that $W=$ $\hat{\mathbf{C}} \backslash A \cup B$ and $u$ is the capacity function for $\operatorname{cap}(A, \hat{\mathbf{C}} \backslash B)$ so that $u \in C(\bar{W}) \cap$ $H(W)$ with $u \mid \alpha=1$ and $u \mid \beta=0$, where $\alpha$ and $\beta$ can also be viewed as being $\alpha=A \cap \partial W$ and $\beta=B \cap \partial W$ with positive directions with respect to $W$. Thus

$$
\operatorname{cap}(A, \hat{\mathbf{C}} \backslash B)=D(u ; W)=D(u ; W \backslash \tau) .
$$

Viewing $W \backslash \tau$ is a subregion of

$$
W_{0}=((\hat{\mathbf{C}} \backslash A \cup B) \backslash \tau)\left(\bigcup_{\tau}(\hat{\mathbf{C}} \backslash \tau)=(W \backslash \tau) \bigotimes_{\tau}(\hat{\mathbf{C}} \backslash \tau) \subset \hat{\mathbf{C}}_{\tau}\right.
$$

$W \backslash \tau$ is a subregion $R$ of $\hat{\mathbf{C}}_{\tau}$ whose relative boundary $\partial R$ consists of $\alpha, \beta$, and $\gamma: \partial R=\alpha+\beta+\gamma$, where $\gamma$ arises from $\tau$ as a smooth Jordan curve positively oriented with respect to $R$ by considering it in the Carathéodory compactification of $R$. Therefore we have

$$
\operatorname{cap}(A, \hat{\mathbf{C}} \backslash B)=D(u ; W \backslash \tau)=D(u ; R) .
$$

By $(2.3) * d u=0$ along $l$ and of course along $\tau$ so that finally along $\gamma$. Restricting $v$ defined on $(W \backslash \tau) \bigotimes_{\tau}(\hat{\mathbf{C}} \backslash \tau)=R \cup \gamma \cup S$ to $R$, where $S:=\hat{\mathbf{C}} \backslash \tau$, we compute the mutual Dirichlet integral $D(u-v, u ; R)$ of two functions $u-v$ and $u$ over $R$ by using the Stokes formula as follows:

$$
D(u-v, u ; R):=\int_{R} d(u-v) \wedge * d u=\int_{\alpha+\beta+\gamma}(u-v) * d u=\int_{\gamma}(u-v) * d u=0
$$

since $* d u=0$ along $\gamma$. Hence $D(u ; R)=D(v, u ; R)$ and the Schwarz inequality yields

$$
D(u ; R)^{2}=D(v, u ; R)^{2} \leq D(v ; R) \cdot D(u ; R) .
$$


Hence we conclude that $D(u ; R) \leq D(v ; R)$ since $D(u ; R)>0$. On the other hand we see that

$$
\begin{aligned}
D(v ; R) & =D(v ; W \backslash \tau)<D(v ; W \backslash \tau)+D(v ; \hat{\mathbf{C}} \backslash \tau) \\
& =D\left(v ; W_{\tau}\right)=D\left(v ;((\hat{\mathbf{C}} \backslash A \cup B) \backslash \tau) \bigotimes_{\tau}(\hat{\mathbf{C}} \backslash \tau)\right)
\end{aligned}
$$

since $D(v ; \hat{\mathbf{C}} \backslash \tau)>0$. The last term of the above is $\operatorname{cap}\left(A, W_{0} \cup A\right)$ so that, by (2.14), we obtain

$$
\operatorname{cap}(A, \hat{\mathbf{C}} \backslash B)<\operatorname{cap}\left(A, W_{0} \cup A\right) .
$$

This with (2.13) we finally conclude that

$$
\operatorname{cap}(A, \hat{\mathbf{C}} \backslash B)<\lim _{t \downarrow 0} \operatorname{cap}\left(A, \hat{\mathbf{C}}_{\gamma_{t}} \backslash B\right)
$$

and therefore we see that

$$
\operatorname{cap}(A, \hat{\mathbf{C}} \backslash B)<\operatorname{cap}\left(A, \hat{\mathbf{C}}_{\gamma_{t}} \backslash B\right)
$$

for every sufficiently small $t \in(0,|\sigma| / 100)$, i.e. $\gamma_{t}$ for sufficiently small $0<t<$ $|\sigma| / 100$ is a supercritical pasting arc for $A$ and $B$ in $\hat{\mathbf{C}}$.

\section{REFERENCES}

[ 1 ] S. Axler, P. Bourdon and W. Ramey, Harmonic function theory, 2nd ed., Springer, 2001.

[2] J. Heinonen, T. KilpeläInen and O. Martio, Nonlinear potential theory of degenerate elliptic equations, Oxford Univ. Press, 1993.

[3] M. NakaI, Types of complete infinitely sheeted planes, Nagoya Math. Jour. 176 (2004), 181-195.

[4] M. NAKaI, Types of pasting arcs in two sheeted spheres, Potential theory in Matsue, to appear in Advanced studies in pure mathematics, 2005.

[5] M. Nakai and S. Segawa, A role of the completeness in the type problem for infinitely sheeted planes, Complex Variables 49 (2004), 229-240.

[6] R. Nevannlina, Analytic functions, Springer, 1970.

[7] B. Rodin AND L. SARIO, Principal functions, Van Nostrand, 1970.

[8] L. SARIO AND M. NAKaI, Classification theory of Riemann surfaces, Springer, 1970.

[9] J. L. SchiFf, Normal families, Springer, 1993.

[10] M. Tsusi, Potential theory in modern function theory, Maruzen, 1959.

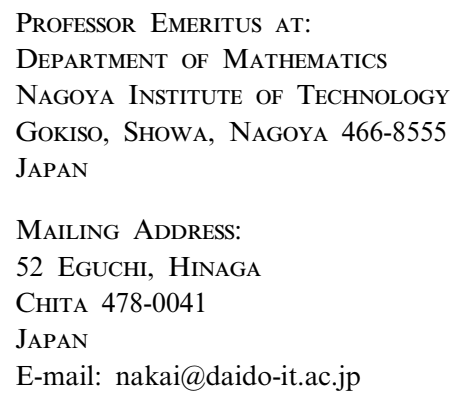

